\title{
Heterogeneidade estrutural: conceito e evidências na América Latina
}

Gabriel Porcile $^{*}$

RESUMO - A heterogeneidade estrutural é uma antiga preocupação das teorias do desenvolvimento. Neste artigo apresentam-se brevemente o marco teórico em que a persistência da heterogeneidade pode ser entendida e algumas evidências para América Latina. Argumenta-se em favor de políticas de mudança estrutural para reduzir a heterogeneidade no longo prazo.

Palavras-chave: heterogeneidade estrutural. mudança tecnológica.

O conceito de heterogeneidade estrutural foi desenvolvido pela CEPAL para explicar porque as economias em desenvolvimento mostram níveis extremamente elevados de subemprego e de assimetrias na produtividade do trabalho, tanto entre setores como no interior dos mesmos. É importante mencionar que diferenças de produtividade são normais e existem em todas as economias. Alguns setores mostram maior dotação de capital e intensidade da inovação; a produtividade é, portanto, maior e tende a crescer a taxas mais altas do que no resto da economia. A geração de vantagens via inovação e oligopólios é normal no capitalismo (SCHUMPETER, 1934). Mas o que singulariza as economias em desenvolvimento é o fato de que a magnitude das assimetrias de produtividade é muito mais elevada e persistente, e atinge a uma maior parcela da força de trabalho (PINTO, 1965, 1970, 1978; RODRIGUEZ, 2007; INFANTE e SUNKEL, 2009; CIMOLI and PORCILE, 2009).

Como se origina a heterogeneidade? Inicialmente, existem duas economias idênticas. Num certo momento (por razões que não é o caso discutir, mas nas quais, como dizem os economistas, a história importa), uma delas acelera sua taxa de inovação, o que se traduz em mudança estrutural e diversificação produtiva. A tecnologia gradualmente se difunde ao conjunto do sistema de forma que surge uma economia homogênea (níveis similares de produtividade do trabalho) e diversificada (existem numerosos setores ou ramas produtivas).

$\mathrm{Na}$ outra economia, o progresso técnico penetra de forma muito parcial e apenas nos setores mais vinculados à exportação. A estrutura que emerge nesse contexto é heterogênea (partes importantes do emprego permanecem em níveis próximos à subsistência)

\footnotetext{
* Mestre em Ciências Econômicas pela Universidade Estadual de Campinas e PhD pela London School of Economics. Atualmente é Professor Associado do Departamento de Economia da Universidade Federal do Paraná (UFPR) e Pesquisador do CNPq. Endereço eletrônico: porcile@ufpr.br.
} 
e especializada (mínima densidade e integração da matriz produtiva). Essa economia não será capaz de gerar o impulso dinâmico necessário para que se difunda o progresso técnico e para que se gerem empregos em atividades de mais alta produtividade -- que possam eventualmente eliminar a heterogeneidade.

A primeira economia (homogênea e diversificada) é o Centro; a segunda (heterogênea e especializada) é a Periferia. A origem das duas estruturas radica nas diferentes taxas de inovação e difusão de tecnologia - e por trás delas, em diferenças políticas e institucionais. O Gráfico 1 ilustra essas estruturas. No Gráfico 1A se observa que a estrutura do centro tem diversos setores (setores de $\mathrm{N}=1$ até $\mathrm{N}=\mathrm{N}^{\mathrm{C}}$ ) e a produtividade muda de forma suave entre eles. A Periferia tem poucos setores (de $\mathrm{N}=1$ até $\mathrm{N}^{\mathrm{P}}$, onde $\mathrm{N}^{\mathrm{C}}>\mathrm{N}^{\mathrm{P}}$ ) e há saltos significativos, degraus qualitativos de produtividade.

GRÁFICO 1A - PRODUTIVIDADE DO TRABALHO E ESTRUTURA

Centro

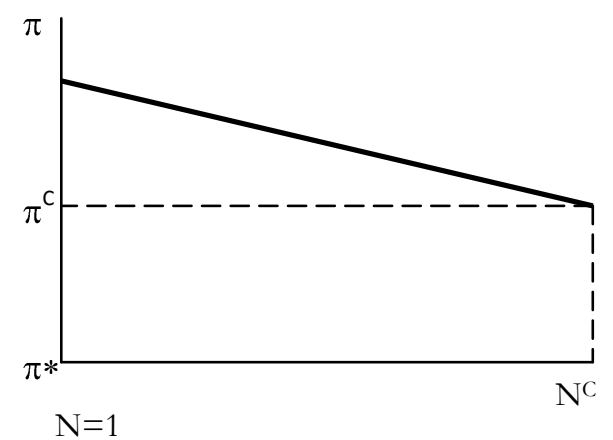

Periferia

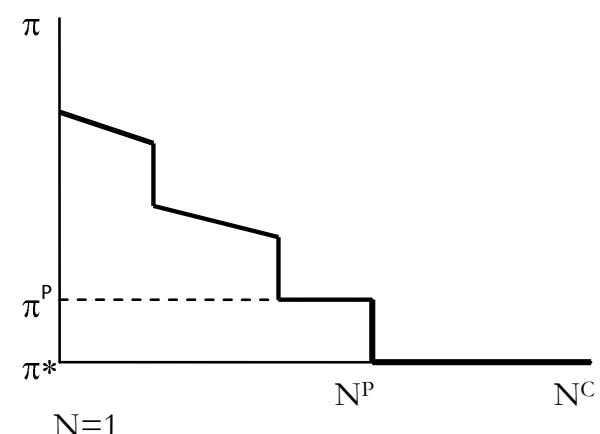

GRÁFICO 1B - EMPREGO E DIVERSIFICAÇÃO

Centro

Periferia

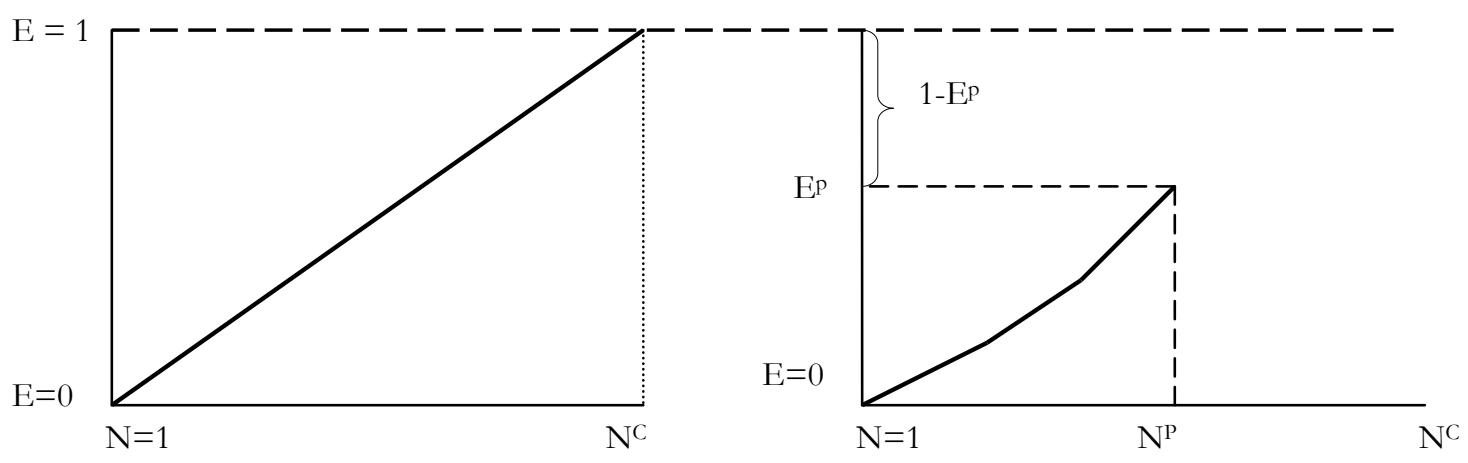

VARIÁVEIS

$\pi=$ Produtividade do trabalho

$\mathrm{E}=$ Participação acumulada no emprego

$\mathrm{N}=$ Número de setores da economia ordenados segundo níveis decrescentes de produtividade do trabalho

$\mathrm{NC}^{\mathrm{C}}=$ Total de setores no centro

$\mathrm{N}^{\mathrm{P}}=$ Total de sectores na periferia

$\mathrm{E}^{\mathrm{P}}=$ Total do emprego nos setores modernos da periferia (o emprego residual 1- $\mathrm{E}^{\mathrm{s}}$ se aloca na subsistência). 
No Gráfico $1 \mathrm{~B}$ se observa a taxa acumulada de emprego (E) por setor: todo o emprego é absorvido nos setores modernos no Centro $\left(E=1\right.$ até $\left.\mathrm{N}^{\mathrm{C}}\right)$, mas na periferia os setores modernos só absorvem uma parte do emprego total, o resto sendo alocado na subsistência $\left(1-\mathrm{E}^{\mathrm{P}}\right)$.

A seguinte tabela, elaborada por Mario Cimoli e Giovanni Stumpo da CEPAL, compara os níveis de heterogeneidade na América Latina e nos Estados Unidos (Tabela 1). Observa-se que:

i) O coeficiente de variação da produtividade do trabalho entre setores é muito mais alto na América Latina que nos Estados Unidos;

ii) Esse coeficiente permanece mais ou menos constante na América Latina e declina um pouco nos Estados Unidos

iii) A dispersão relativa da produtividade entre América Latina e os Estados Unidos aumenta entre 1990 e 2008.

O Gráfico 1 confirma essa maior dispersão relativa da produtividade na América Latina e aponta ainda um fenômeno preocupante, qual seja a diferença crescente entre a produtividade média do trabalho nos Estados Unidos e na América Latina. Assim como existe um hiato de produtividade crescente entre países, também se observa o aumento do hiato entre trabalhadores da América Latina.

O tema da heterogeneidade estrutural está recebendo uma atenção cada vez maior por parte dos governos da região. Algumas medidas procuram facilitar o acesso das pequenas e médias empresas, e dos setores atrasados, à tecnologia. Também (ainda timidamente) procura-se qualificar aos trabalhadores. Mas os dados indicam que uma absorção dinâmica do subemprego exige taxas mais elevadas de crescimento e políticas tecnológicas que estimulem o adensamento da matriz produtiva.

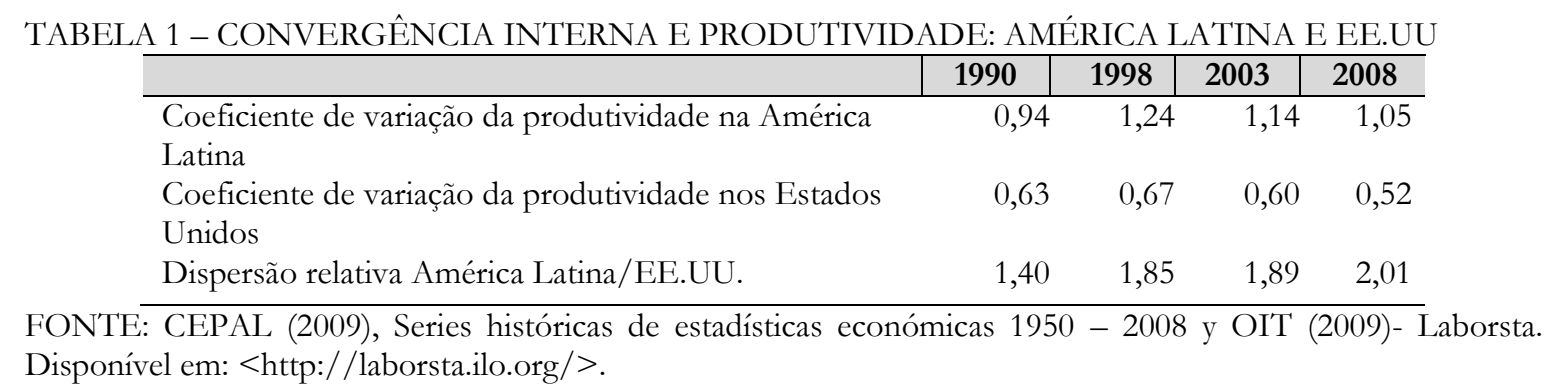

Disponível em: <http://laborsta.ilo.org/>. 
GRÁFICO 1 - PRODUTIVIDADE RELATIVA AMÉRICA LATINA/EE.UU. E COEFICIENTE DE DISPERSÃO DA MESMA

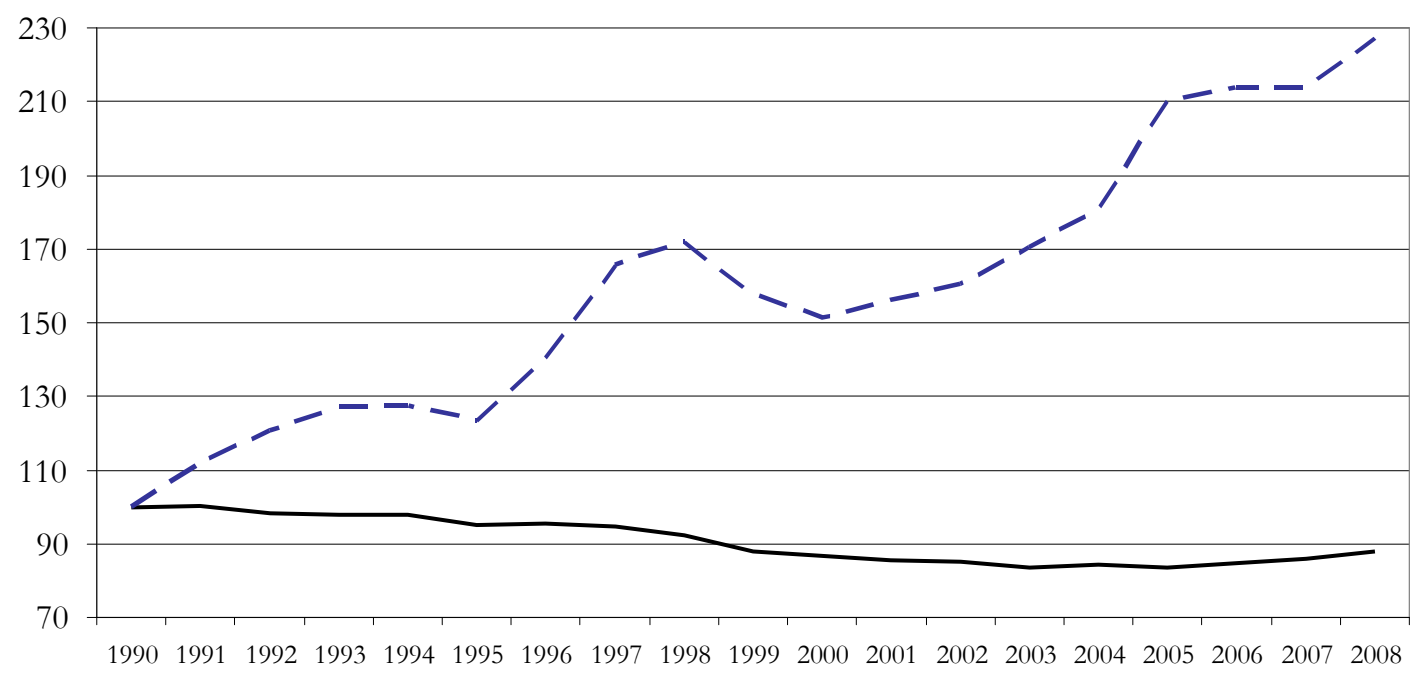

$$
\text { - PRD relativa - Coef dispersão }
$$

FONTE: CEPAL (2009), Series históricas de estadísticas económicas 1950 - 2008 y OIT (2009)- Laborsta. Disponível em: <http://laborsta.ilo.org/>.

\section{REFERÊNCIAS}

CEPAL (Comisión Económica para América Latina y el Caribe), América Latina y el Caribe: Series históricas de estadísticas económicas 1950-2008, Cuadernos Estadísticos, No 37 (LC/G.2415-P), Comisión Económica para América Latina y el Caribe (CEPAL), Santiago de Chile

CEPAL, PROGRESO TÉCNICO Y CAMBIO ESTRUCTURAL EN AMÉRICA LATINA, Documento de Proyecto, LC/W136, Comisión Económica para América Latina y el Caribe (CEPAL), Santiago de Chile.

CIMOLI, M. and PORCILE, G. Sources of Learning Paths and Technological Capabilities: An Introductory Roadmap of Development Processes, Economics of Innovation and New Technology, 1476-8364, Volume 18, Issue 7, 2009, Pages 675- 694

INFANTE R. y O. SUNKEL, Chile: hacia un desarrollo inclusivo, Revista de la CEPAL, No 97, Comisión Económica para América Latina y el Caribe (CEPAL), Santiago de Chile.

OIT - Laborsta Internet. Disponível em: <http://laborsta.ilo.org>.

PINTO, A. Notas sobre los estilos de desarrollo en América Latina, Revista de la CEPAL, $\mathrm{N}^{\circ}$ 1, Santiago de Chile, primer semestre

PINTO, A. Naturaleza e implicaciones de la heterogeneidad estructural de la America Latina. FCE, México.

PINTO, A. Concentración del progreso técnico y de sus frutos en el desarrollo latinoamericano. Trimestre Económico. Enero-marzo. FCE, México.

RODRÍGUEZ, O. E1 Estructuralismo Latinoamericano. México: Siglo XXI.

SCHUMPETER, J.A. The Theory of Economic Development, Cambridge, Harvard University Press. 